\title{
Time Domain Measures of Heart Rate Variability to Assess Autonomic Dysfunction In Irritable Bowel Syndrome
}

\author{
Mohammad Nayem ${ }^{1}$, Noorzahan Begum ${ }^{2}$, Sultana Ferdousi ${ }^{3}$
}

\begin{abstract}
Background: Autonomic nerve function impairment is related to development of Irritable Bowel Syndrome (IBS). Time domain measures of Heart rate variability (HRV) is a useful tool to measure autonomic nerve function activity. Objective: To assess autonomic nerve function activity by time domain measures of heart rate variability in patients with Irritable Bowel Syndrome. Methods: This cross sectional study was conducted in the Department of Physiology, Bangabandhu Sheikh Mujib Medical University (BSMMU), Dhaka in 2011. Ninety patients aged 20-50 years of both sex with Irritable bowel syndrome were included in the study group. They were collected from the OPD of Gastroenterology in BSMMU. Age and sex matched 30 apparently healthy subjects served as control. For assessing HRV by time domain method, Mean heart rate Mean R-R interval, Max/Min R-R interval, SDNN, RMSSD, PNN50\%, NN50\% were recorded by a digital Polyrite. ANOVA, independent sample t-test and Pearson's correlation coefficient tests were performed as applicable. Results: Mean heart rate were significantly higher and Mean R-R interval, Max/Min R-R interval, SDNN, RMSSD, PNN50\%, NN50\% were significantly lower in IBS groups compared to those of control. Correlation analysis showed negative correlations of SDNN, RMSSD, PNN50\%, NN50\% with duration of disease.
\end{abstract}

Conclusion: This study concludes that parasympathetic activity was reduced in patients of IBS. In addition, decreased vagal modulation is inversely related to the duration IBS.

Key words: Irritable bowel syndrome, Autonomic nerve function, Heart rate variability.

\author{
J Bangladesh Soc Physiol. 2012 December; 7(2): 60-65 \\ For Authors Affiliation, see end of text. \\ http://www.banglajol.info/index.php/JBSP
}

\section{Introduction \\ I rritable bowel syndrome (IBS) is a chronic continuous or remittent functional gastro- intestinal disorder, characterized by abdominal pain, bloating and bowel disturbance. ${ }^{1}$ IBS constitutes the major bulk of gastrointestinal tract related health problem. The prevalence of IBS was found $24.4 \%$ in rural and $7.7 \%$ in urban community of Bangladesh with male female ratio $1: 1.36^{2}$}

Received; June 2012 Accepted : September 2012
Three subgroups of IBS patients were defined on the basis of Rome supportive symptoms. It includes diarrhea predominant IBS (IBS-D), constipation predominant IBS (IBS-C), and IBS with alternate bowel habit (IBS-A). ${ }^{2}$

On the basis of abdominal and bowel symptoms IBS is diagnosed and classified following revised Rome-II criteria. ${ }^{3}$ The criteria for diagnosis of IBS include abdominal discomfort or pain where at least two of the features are to be present which is relieved by defecation, onset is associated with 
a change in frequency and form of stool. The symptom must persist at least 12 weeks, in the preceding one year. ${ }^{2}$

IBS is suggested to be a multifactorial in origin. Several environmental factors, psycho social stressors, altered gut flora contribute to pathophysiology of IBS. It is now largely considered as a disorder of the brain-gut axis, involving abnormal function in the enteric, autonomic and central nervous system. ${ }^{4}$

Autonomic nervous system regulates the visceral sensitivity of the body and coordinates gastrointestinal motility and secretion. ${ }^{5}$ Recently it was proposed that autonomic nervous system may be affected by inflammatory reaction at the level of enteric mucosa. ${ }^{6}$

Heart rate variability (HRV) is a measure of autonomic nervous control of the body. Normally HR variation is related to the balance between sympathetic and parasympathetic nervous system which provides better qualitative and quantitative interpretation of sympathovagal modulation and can detect autonomic impairment ${ }^{10}$. Higher HRV is assumed to indicate good adaptability and well functioning autonomic control. On the other hand, its reduction serves as a strong predictor of risk for adverse events with wide range of diseases. ${ }^{7}$ Usually simple time domain like mean heart rate, mean R-R interval, Max/Min R-R interval and statistical time domain like SDNN, RMSSD, PNN50\% and NN50\% are used for HRV measurement. ${ }^{8}$

Significantly lower mean R-R interval was observed in IBS patients compared to the healthy control. ${ }^{9}$ However, higher mean heart rate was also reported.$^{10}$

SDNN, RMSSD and PNN50\% were found to be variable in different clinical form of IBS. ${ }^{11,12}$ Lower SDNN value was reported in IBS-C. ${ }^{11}$ Whereas another group demonstrated lower value of SDNN, RMSSD, PNN50\% of the similar groups of patients compared to the controls. ${ }^{12}$ Interestingly higher values of all these variables were observed in IBS-D but only higher RMSSD and $\mathrm{PNN} 50 \%$ in IBS-C compared to the controls. ${ }^{11}$
Relatively high prevalence of IBS in Bangladesh has been reported in both rural and urban area which deserves greater attention. Although number of studies by different investigators have been attempted but pathological basis of IBS yet to be clearly understood in particular the autonomic nervous system involvement. Autonomic nerve function evaluated in athletes, subjects with sedentary life and patients of hypothyroidism and hyperthyroidism and also rheumatoid arthritis. (13-17) However data regarding autonomic nerve function of IBS patients are lacking. Hence, in the present study was aimed to evaluate autonomic nerve function in IBS patients to explore any difference in different IBS subtypes and also to assess the importance of these test in the clinical practice.

\section{Methods}

This cross sectional study was conducted in the Department of Physiology, Bangabandhu Sheikh Mujib Medical University (BSMMU), Shahbag, Dhaka from July 2010 to June 2011. Study subjects were selected by following simple random sampling procedure and the protocol of this study was approved by central ethical review committee of BSMMU. Total number of 90 patients of Irritable Bowel Syndrome (45 male and 45 female) age 2050 years, were enrolled in the study group (Group B). According to Rome supportive symptoms criteria based on bowel habit predominance, IBS patients were subdivided into, Group $\mathrm{B}_{1}(\mathrm{n}=30)$ having symptoms of diarrhea predominance IBS (IBS-D), Group $\mathrm{B}_{2}(\mathrm{n}=30)$ with constipation predominance IBS (IBS-C) and Group $\mathrm{B}_{3}(\mathrm{n}=30)$ with alternate bowel habits IBS (IBS-A). Age and sex matched 30 apparently healthy subjects (Group A) was served as control group. The patients were collected from out patient Department of Gastroenterology, (BSMMU), Dhaka and the healthy subjects of control group was recruited through personal contact. IBS patients with history of heart diseases, hypertension, diabetes mellitus and chronic renal failure, habit of smoking, pregnancy and thyroid disorder were excluded from the study.

Selected subjects were informed about the risk and benefit and detail procedure of the study 
before enrollment and written consent was obtained from the willing volunteers. For recording HRV parameters subjects were prepared from the preceding day of examination. They were advised to finish their meal by 9:00 pm on the previous night, to remain free from any physical or mental stress, not to take sedatives or any drugs affecting nervous system and to have a sound sleep at night. The subjects were also asked to have light breakfast without tea or coffee. All examinations were done in the Autonomic Nerve Function Test Laboratory in the Department of Physiology, BSMMU between 9a.m. to 1 p.m. Autonomic nerve function test of all subjects were done by time domain mesures of HRV. For Autonomic Nerve Function Test the subject lied on a bed in supine position and allowed to take rest for 15-20 minutes. 5 minutes ECG recording for HRV was taken by a polygraph. Time domain measures of
HRV like Mean heart rate, Mean R-R interval, Max/Min R-R interval, SDNN, RMSSD, PNN50\%, NN50\% were analyzed. ANOVA and unpaired ttests were done as applicable. $\mathrm{P}$ value $<0.05$ was taken as level of significance.

\section{Results}

All the groups were matched for age and BMI.

Mean values of mean heart rate were significantly higher and Mean R-R interval, Max/Min R-R interval were significantly lower in group B1, B2 and B3 than that of group A. (Table I)

The mean values of SDNN, RMSSD, PNN50\%, and NN50\% were significantly lower in group B1, B2 and B3 than that of group A. (Table II)

There was no significant difference in all measures when compared between B1 vs B2, B2 vs $\mathrm{B} 3$ and $\mathrm{B} 3$ vs $\mathrm{B} 1$.

Table I: Simple time domain measures of HRV in different groups $(n=120)$

\begin{tabular}{|c|c|c|c|}
\hline Groups & Mean heart rate(beat/min) & Mean R-R interval(sec) & $\overline{\text { Max/Min R-Rinterval }}$ \\
\hline$\overline{A(n=30)}$ & $78 \pm 7.15(65-90)$ & $0.78 \pm 0.10(0.60-1.00)$ & $1.98 \pm 0.83(1.01-5.32)$ \\
\hline $\mathrm{B} 1(\mathrm{n}=30)$ & $87 \pm 10.54(71-125)$ & $0.65 \pm 0.10(0.48-1.00)$ & $1.64 \pm 0.44(1.08-2.71)$ \\
\hline $\mathrm{B} 2(\mathrm{n}=30)$ & $86 \pm 10.31(65-109)$ & $0.67 \pm 0.10(0.50-0.86)$ & $1.58 \pm 0.36(1.02-2.59)$ \\
\hline $\mathrm{B} 3(\mathrm{n}=30)$ & $88 \pm 12.74(62-110)$ & $0.64 \pm 0.11(0.37-0.86)$ & $1.60 \pm 0.37(1.14-2.69)$ \\
\hline \multicolumn{4}{|l|}{ Statistical analysis } \\
\hline Groups & $p$ values & & \\
\hline$\overline{\mathrm{A} \text { vs B1 vs B2 vs.B3 }}{ }^{\mathrm{a}}$ & $0.000^{* * *}$ & $0.000^{* * *}$ & $0.007^{* *}$ \\
\hline A vs.B1 ${ }^{b}$ & $0.000^{* * *}$ & $0.000^{* * *}$ & $0.037^{*}$ \\
\hline A vs.B2 ${ }^{b}$ & $0.000^{* * *}$ & $0.000^{* * *}$ & $0.011^{*}$ \\
\hline A vs.B3 ${ }^{b}$ & $0.000^{* * *}$ & $0.000^{* * *}$ & $0.015^{*}$ \\
\hline $\mathrm{B} 1$ vs.B2 ${ }^{\mathrm{b}}$ & $0.727^{\mathrm{ns}}$ & $0.466^{\mathrm{ns}}$ & $0.511^{\mathrm{ns}}$ \\
\hline $\mathrm{B} 2$ vs.B $3^{\mathrm{b}}$ & $0.413^{\mathrm{ns}}$ & $0.270^{\mathrm{ns}}$ & $0.852^{\mathrm{ns}}$ \\
\hline $\mathrm{B} 3 \mathrm{vs.B}^{\mathrm{b}}$ & $0.616^{\mathrm{ns}}$ & $0.691^{\mathrm{ns}}$ & $0.629^{\mathrm{ns}}$ \\
\hline
\end{tabular}

Data were expressed as Mean \pm SD. Figures in parentheses indicate range.

Statistical analysis were done by One-way ANOVA ${ }^{\mathrm{a}}$ and Independent sample t-test ${ }^{\mathrm{b}}$

$\mathrm{R}-\mathrm{R}$ = Interval between successive QRS complex ( $\mathrm{sec}$ ),

Max = Maximum,

Min = Minimum.

Group A: Apparently healthy subjects (control)

Group B1: Diarrhoea predominant Irritable Bowel Syndrome (IBS-D)

Group B2: Constipation predominant Irritable Bowel Syndrome (IBS-C)

Group B3: Irritable Bowel Syndrome with alternate bowel habit (IBS-A)

$* * *=\mathrm{p}<0.001 \mathrm{~ns}=$ non significant $(\mathrm{p}>0.05)$

$* *=\mathrm{p}<0.01 \quad \mathrm{n}=$ number of subjects.

$* \quad=p<0.05$

J Bangladesh Soc Physiol. 2012, December; 7(2): 60-65 
Table II: Statistical time domain measures of HRV in different groups $(n=120)$

\begin{tabular}{lcccc}
\hline Groups & $\begin{array}{c}\text { SDNN } \\
(\mathrm{ms})\end{array}$ & $\begin{array}{c}\text { RMSSD } \\
(\mathrm{ms})\end{array}$ & $\begin{array}{c}\text { NN50 } \\
(\%)\end{array}$ & $\begin{array}{c}\text { PNN50 } \\
(\%)\end{array}$ \\
\hline $\mathrm{A}(\mathrm{n}=30)$ & $74.95 \pm 18.40$ & $34.30 \pm 4.39$ & $71 \pm 30.30$ & $24.4 \pm 14.75$ \\
& $(20.40-98.70)$ & $(22.54-40.23)$ & $(25-126)$ & $(7.0-55.6)$ \\
$\mathrm{B} 1(\mathrm{n}=30)$ & $49.38 \pm 15.26$ & $18.81 \pm 5.85$ & $19 \pm 9.79$ & $9.2 \pm 5.46$ \\
& $(22.55-92.68)$ & $(10.21-29.64)$ & $(6-45)$ & $(2.3-21.0)$ \\
$\mathrm{B} 2(\mathrm{n}=30)$ & $41.76 \pm 18.70$ & $21.05 \pm 8.79$ & $20 \pm 11.26$ & $10.4 \pm 4.09$ \\
& $(11.67-78.51)$ & $(10.92-36.54)$ & $(7-51)$ & $(3.5-18.0)$ \\
$\mathrm{B} 3(\mathrm{n}=30)$ & $46.82 \pm 19.59$ & $22.07 \pm 8.77$ & $23 \pm 9.65$ & $10.9 \pm 6.83$ \\
& $(21.79-87.47)$ & $(10.77-44.55)$ & $(9-42)$ & $(3.2-28.8)$ \\
\hline
\end{tabular}

Statistical analysis

\begin{tabular}{|c|c|c|c|c|}
\hline \multirow{2}{*}{$\frac{\text { Groups }}{\text { Avs B1vs B2vs.B3 }}$} & \multicolumn{4}{|c|}{$\mathrm{p}$ values } \\
\hline & $0.000^{* * *}$ & $0.000^{* * *}$ & $0.000^{* * *}$ & $0.000^{* * *}$ \\
\hline A vs.B $1^{b}$ & $0.000^{* * *}$ & $0.000^{* * *}$ & $0.000^{* * *}$ & $0.000^{* * *}$ \\
\hline A vs.B $2^{b}$ & $0.000^{* * *}$ & $0.000^{* * *}$ & $0.000^{* * *}$ & $0.000^{* * *}$ \\
\hline A vs.B $3^{b}$ & $0.000^{* * *}$ & $0.000^{* * *}$ & $0.000^{* * *}$ & $0.000^{* * *}$ \\
\hline B1 vs.B2 b & $0.072^{\mathrm{ns}}$ & $0.206^{\mathrm{ns}}$ & $0.757^{\mathrm{ns}}$ & $0.328^{\mathrm{ns}}$ \\
\hline B2 vs.B3 b & $0.287^{\mathrm{ns}}$ & $0.230^{\mathrm{ns}}$ & $0.139^{\mathrm{ns}}$ & $0.713^{\mathrm{ns}}$ \\
\hline $\mathrm{B} 3$ vs.B $1^{\mathrm{b}}$ & $0.552^{\mathrm{ns}}$ & $0.067^{\mathrm{ns}}$ & $0.062^{\mathrm{ns}}$ & $0.273^{\mathrm{ns}}$ \\
\hline
\end{tabular}

Data were expressed as Mean $\pm \mathrm{SD}$. Figures in parentheses indicate range.

Statistical analysis were done by One-way ANOVA ${ }^{\mathrm{a}}$ and Independent sample t-test ${ }^{\mathrm{b}}$

SDNN $\quad=$ Standard deviation of NN interval

RMSSD $=$ Square root of mean squared differences between adjacent NN intervals

$\mathrm{NN} 50 \%=$ number of interval differences of successive NN intervals greater than $50 \mathrm{~ms}$

PNN50\% = number of R-R interval differing by $>50 \mathrm{~ms}$ from adjacent intervals divided by the total number of all R-R intervals

ms $\quad=$ millisecond

Group A: Apparently healthy subjects (control)

Group B1: Diarrhoea predominant Irritable Bowel Syndrome (IBS-D)

Group B2: Constipation predominant Irritable Bowel Syndrome (IBS-C)

Group B3: Irritable Bowel Syndrome with alternate bowel habit (IBS-A)

$* * *=\mathrm{p}<0.001 \quad \mathrm{~ns}=$ non significant $(\mathrm{p}>0.05)$

$* *=\mathrm{p}<0.01 \quad \mathrm{n}=$ number of subjects.

$* \quad=p<0.05$

\section{Discussion}

In last decade several investigators had assessed autonomic activity by time domain measures of HRV from the ECG recording in IBS patients. The present study was undertaken to observe the HRV in IBS patients in order to assess their autonomic nervous activity.
In the present study, values of HRV parameters in healthy subjects were almost within normal range and also similar to those reported by the several investigators from abroad $9-12,18$ and from our country. ${ }^{13-17}$

In this study, significantly higher values of mean heart rate represents higher sympathetic activity 
in IBS patients irrespective of subgroups. Similar types of findings were also reported by the various investigators of different countries. ${ }^{9}$ But the results did not show any significant difference in sympathetic activity among the subtypes of IBS patients.

Mean R-R interval, Max/Min R-R interval, SDNN, RMSSD, PNN50\%, NN50\% were significantly lower in IBS patients which indicate lower parasympathetic activity which is consistent with others. ${ }^{11,12}$ Similar trend was also observed by the various investigators of different countries. ${ }^{11,12}$ But the results did not discriminate parasympathetic activity among the subtypes of IBS patients. Lack of differences among the subtypes of IBS patients may be explained by the predominance of autonomic enteric nervous system activity. ${ }^{3}$

Different investigators explained various mechanisms for involvement of autonomic nerve function activity in IBS patients. One of the predispositions was increased release of corticotrophin releasing factor (CRF) from hypothalamus which may affect directly or through locus ceruleus. It is postulated to excite anxiety and bowel motility.$^{10}$

It is understood that anxiety, depressive disorder and emotional state cause lower parasympathetic nervous system activity which is supposed to alter autonomic nervous system input to the gastrointestinal system. ${ }^{12}$ It has also been suggested that this autonomic nerve dysfunction may influence the descending inhibitory pathway and increase bowel sensitivity.

The present study demonstrated increased sympathetic activity and reduced vagal tone in IBS patients. Again, significant changes of all the HRV parameters indicate shifting of sympathovagal balance towards sympathetic predominance. These features represent autonomic dysfunction of IBS patients of present series. This dysfunction is most likely attributed to anxiety, depressive disorder, somatization disorder, psychological distress and emotional factors. However attempts were made to circumvent anxiety, psychological distress and emotional factors by making patients comfortable during the procedure. In case of screening of the subjects for subclinical depressive disorders conclusive comments could have been made regarding their autonomic nerve dysfunction.

\section{Conclusions}

It is concluded that IBS patients have markedly decreased parasympathetic but increased sympathetic nerve function status and different forms of IBS showed similar pattern of autonomic nerve dysfunction.

\section{Author affiliations}

1. *Mohammad Nayem, Assistant professor, Department of Physiology, Dhaka National Medical College, 53/1 Johnson Road, Dhaka-1100. Email: mohammadnayem7@gmail.com

2. Noorzahan Begum, Professor, Department of Physiology, Bangabandhu Sheikh Mujib Medical University(BSMMU) Bangladesh. Email:noorzahan 52@gmail.com

3. Sultana Ferdousi, Associate professor, Department of Physiology, Bangabandhu Sheikh Mujib Medical University(BSMMU) Bangladesh. Email: sferdousi ratna@gmail.com

*For correspondence

\section{References}

1. Clark C, DeLegge M. Irritable Bowel Syndrome: A Practical Approach. Nutr Clin Pract. 2008; 23:263267.

2. Perveen I, Hasan M, Masud MA, Rahman MM, Bhuiyan MMR. Irritable Bowel Syndrome In A Bangladeshi Urban Community: Prevalance And Health Care Seeking Pattern. Saudi J Gastroentrology.2009; 15(4):239-43.

3. Karantanos T, Markoutsaki T, Gazouli M, Anagnou NP, Karamanolis DG. Current Insights Into The Pathophysiology Of Irritable Bowel Syndrome.Gut Pathogens. 2010, 2:3.

4. Posserud I, Ersryd A, Simren M. Functional Findings In Irritable Bowel Syndrome.World J Gastroentrol. 2006; 12:2830-2838.

5. Elenkov U, Wilder RL, Chrousos GP. The Sympathetic Nerve-An Integrative Interface Between Two Supersystems: The Brain And 
Immune System. Pharmacol Rev. 2000; 52: 585638 .

6. Hansen MB. The Enteric Nervous System I: Organization And Classification. Pharmacol Toxicol. 2003; 92: 105-113.

7. Evrengül H, Dursunoglu D, Cobankara V, Polat B, Seleci D, Kabukeu S, Kaftan A, Semiz E, Kilic M. Heart Rate Variability In Patients With Rheumatoid Arthritis. Rheumatol Int.2004; 24:198-202.

8. Task Force Of The European Society Of Cardiology And The North American Society Of Pacing And Electrophysiology, Heart Rate Variability. Standards Of Measurement, Physiological Interpretation And Clinical Use. Circulation. 1996; 93: 1043- 65.

9. Aggarawal A, Cutts TF, Abell TL, Cardoso S, Familoni B, Bremer J, Karas J. Predominant Symptoms In Irritable Bowel Syndrome Correlate With Specific Autonomic Nervous Abnormalities. Gastroenterology 1994; 106(4): 945-950.

10. Karling P, Nyhlin H, Wikilund U, Sjoberg M .Olofsson BO, Bjerle P. Spectral Analysis Of Heart Rate Variability In Patients With Irritable Bowel Syndrome. Scand J Gastroenterol.1998; 3: 572576.

11. Heitkemper M, Jarrett M, Cain KC, Burr R, Levy RL, Feld A, Hertig V. Autonomic Nervous System Function In Women With Irritable Bowel Syndrome. Digestive Disease and Sciences.2001; 46(6):1276-1284
12. Jarrett ME, Burr RL, Cain KC, Hertig V, Weisman P, Arnp M, Heitkemper MM. Anxiety and Depression Are Related To Autonomic Nervous System Function In Women With Irritable Bowel Syndrome. Digestive Diseases and Sciences .2003; 48(2):386-394.

13. Kabir MR. Study On Heart Rate Variability In Patients With Hyperthyroidism.[Thesis] [Dhaka(Bangladesh)]:BSMMU.2008

14. Alom M. Study On Heart Rate Variability In Adloscent Male Athlete [Thesis] [Dhaka ((Bangladesh)]:BSMMU.2008 July.

15. Mithun S. Comparative Study Of Cardiac Autonomic Nerve Function Status By Heart Rate Variability Between Sedentary And Heavy Workers. [Thesis][Dhaka (Bangladesh)]:BSMMU.2006 July.

16. Tabassum R. Assessment Of Cardiac Autonomic Nerve Function Status By Heart Rate Variability In Essential Hypertension.[Thesis] [Dhaka (Bangladesh)]: BSMMU.2008 July.

17. Jahan K. Assessment Of Cardiac Autonomic Nerve Function Status In Patients With Rheumatoid Arthritis. [Thesis][Dhaka(Bangladesh)] : BSMMU.2011.

18. Robert JJT, Elsenbruch S, Whitehead WC. Sleep Related Autonomic Disturbances In Symptom Subgroups Of Women With Irritable Bowel Syndrome. Dig Dis Sci. 2006; 51:2121-2127. 